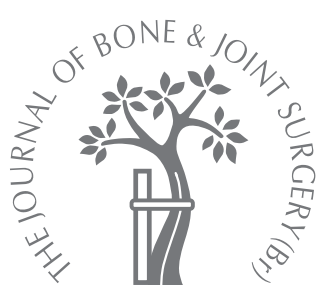

M. Ozbaydar,

B. Elhassan,

C. Esenyel,

A. Atalar,

E. Bozdag,

E. Sunbuloglu,

N. Kopuz,

M. Demirhan

From Istanbul School

of Medicine,

Istanbul, Turkey

M. Ozbaydar, MD,

Orthopaedic Surgeon

Department of Orthopaedics

and Traumatology

Okmeydani Teaching Hospital,

34387, Okmeydani, Istanbul,

Turkey.

B. Elhassan, MD, Assistant

Professor

Mayo Clinic, 200 First Street,

Rochester, Minnesota 55905,

USA.

․ Esenyel, MD, Orthopaedic

Surgeon

Department of Orthopaedics

and Traumatology

Vakif Gureba Teaching Hospital,

34093, Fatih, Istanbul, Turkey.

A. Atalar, MD, Orthopaedic

Surgeon

M. Demirhan, MD, Professor

Department of Orthopaedics

and Traumatology

Istanbul University, Istanbu

School of Medicine, 34390,

Capa, Istanbul, Turkey.

E. Bozdag, PhD, Researcher

E. Sunbuloglu, MSc,

Researcher

Department of Medica

Engineering

Istanbul Technical University,

34437, Güssüssuyu, Istanbul,

Turkey.

N. Kopuz, MD, Pathologis Department of Pathology

Istanbul University,

Cerrahpasa Medical Faculty,

34098, Fatih, Istanbul, Turkey.

Correspondence should be sent to Dr M. Ozbaydar; e-mail: mehmetozbaydar@hotmail.com

(C)2008 British Editorial Society of Bone and Joint Surgery doi:10.1302/0301-620X.90B10. doi: $10.1302 / 032 \$ 2.00$

$J$ Bone Joint Surg [Br] 2008;90-B:1386-91.

Received 4 February 2008

Accepted after revision 15 May

2008

\title{
A comparison of single- versus double-row suture anchor techniques in a simulated repair of the rotator cuff
}

\author{
AN EXPERIMENTAL STUDY IN RABBITS
}

\begin{abstract}
We compared time-dependent changes in the biomechanical properties of single- and double-row repair of a simulated acute tear of the rotator cuff in rabbits to determine the effect of the fixation techniques on the healing process.

A tear of the supraspinatus tendon was created in 80 rabbits which were separated into two equal groups. A single-row repair with two suture anchors was conducted in group 1 and a double-row repair with four suture anchors in group 2. A total of ten intact contralateral shoulder joints was used as a control group. Biomechanical testing was performed immediately post-operatively and at four and eight weeks, and histological analysis at four and eight weeks.
\end{abstract}

The mean load to failure in group 2 animals was greater than in group 1, but both groups remained lower than the control group at all intervals. Histological analysis showed similar healing properties at four and eight weeks in both groups, but a significantly larger number of healed tendon-bone interfaces were identified in group 2 than in group 1 at eight weeks (p $<0.012$ ).

The ultimate load to failure increased with the number of suture anchors used immediately post-operatively, and at four and eight weeks. The increased load to failure at eight weeks seemed to be related to the increase in the surface area of healed tendon-tobone in the double-row repair group.

Studies of the normal anatomy of the insertion of the rotator cuff have shown that the 'footprint' of the supraspinatus tendon insertion is extensive. ${ }^{1}$ In order to maximise the healing of a tear of the rotator cuff, the technique of double-row suture anchor fixation creates a significantly larger contact area and greater overall pressure distribution than techniques of transosseous tunnel repair and single-row suture anchor methods. ${ }^{2-4}$ It has also been shown that double-row fixation can improve the initial fixation strength of rotator cuff repairs. ${ }^{5,6}$ Most of the studies performed to date which evaluate the healing of the rotator cuff after repair were based on clinical and imaging studies. However, assessment of the biological healing as well as the biomechanical properties of the rotator cuff after single- and double-row repair has not been undertaken.

The goal of this study was to compare the time-dependent changes in the biomechanical properties of single- and double-row fixation in the repair of a simulated acute rotator cuff tear, and to determine the effect of each technique on the biological healing process in the early postoperative period. The hypothesis was that fixation with the double-row technique would improve early post-operative healing.

\section{Materials and Methods}

The Institutional Animal Care and Use Committee approved the protocol for the study. A total of 80 adult New Zealand white rabbits aged between 24 and 28 months, with a mean body-weight of $3.5 \mathrm{~kg}$ (3 to 3.9) was used. They were housed individually before and after surgery and had unrestricted access to their standard diet and water. The choice of rabbits for this study was based on previous studies that showed them to be appropriate models. ${ }^{7-9}$

Using alternating sides, one shoulder of each rabbit was operated on to create a complete tear of the supraspinatus tendon medial to its insertion on the greater tuberosity. The rabbits were divided randomly into two equal-sized groups with the torn supraspinatus tendon being repaired in 40 animals with a single-row technique using two suture anchors (group 1). The other 40 animals were treated with a double-row technique using four suture anchors (group 2). From each group, 30 rabbits were used for biomechanical testing and 


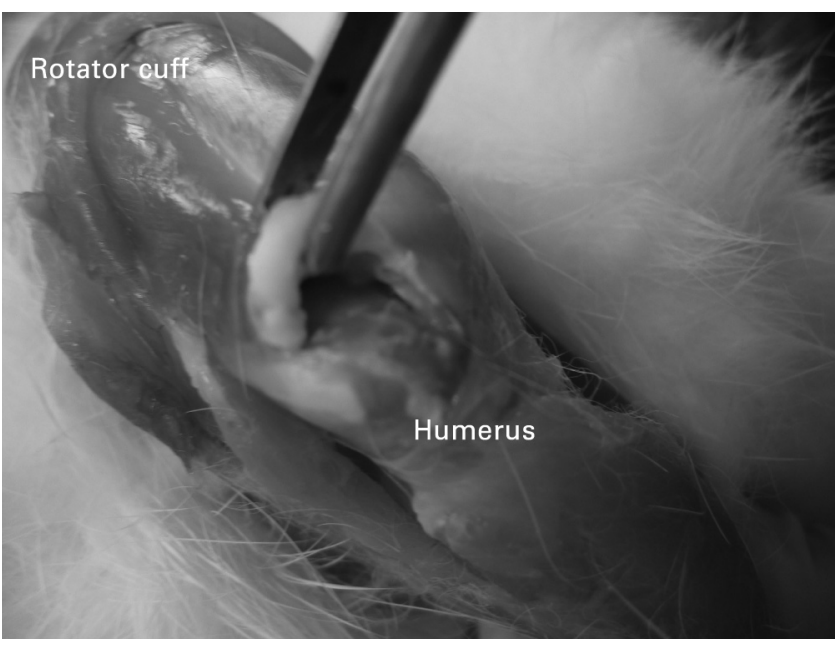

Fig. 1

Photograph showing full-thickness division (tear) of the supraspinatus tendon.

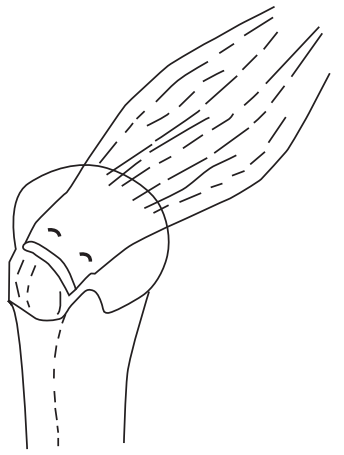

Fig. 2a

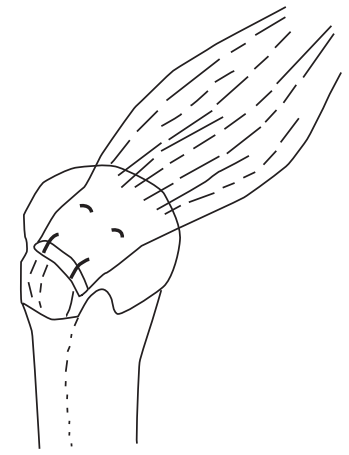

Fig. $2 b$
A drawing of the a) single-row and b) double-row repair techniques.

ten for histological analysis. The unoperated contralateral shoulder joints of ten animals constituted the control group. The 30 rabbits in each group that were used for biomechanical tests were divided into three equal subgroups and biomechanical tests were performed on the ten rabbits in each subgroup immediately after surgery (week 0 ), four weeks after surgery, and eight weeks after surgery. The ten rabbits in each group that were used for histological analysis were divided into two subgroups of five each for histological evaluation at four weeks and eight weeks after surgery.

Operative procedure. Before surgery, ketamine $(50 \mathrm{mg} / \mathrm{kg})$ and xylazine $(5 \mathrm{mg} / \mathrm{kg})$ were injected into the rabbits' hindquarters as premedication, followed by general anaesthesia via inhalation of nitrous oxide and oxygen $\left(\mathrm{N}_{2} \mathrm{O} / \mathrm{O}_{2}=1: 2\right)$ and sevoflurane $(2.5 \%)$. Through a $3 \mathrm{~cm}$ longitudinal anterolateral incision, the omovertebral and deltoid muscles were retracted to expose the supraspinatus tendon, which was then sharply transected over a $10 \mathrm{~mm}$ width close to its insertion into the greater tuberosity to simulate a tear of the rotator cuff. In essence, a full-thickness defect was created on the supraspinatus tendon. The remaining distal fibrocartilaginous stump was resected, exposing the footprint of the supraspinatus tendon (Fig. 1) which was then debrided using a curette. The two repair configurations, comprising a standard single-row anchor repair technique (group 1) and a footprint double-row anchor technique (group 2) were randomised and tested on each set of tendon specimens (Fig. 2) with the repair provided by a $2.4 \mathrm{~mm}$ metal suture anchor (Arthrex, Naples, Florida), single loaded with a polyester braid and long-chain polyethylene suture (number 1 FibreWire; Arthrex). All repairs were performed in an open setting, using an arthroscopic knot-tying technique. The same surgeon (MO) placed and tied all stitches manually to minimise variability. All distances and measurements were carefully performed using calipers to standardise the technique for each shoulder.

Single-row anchor repair (group 1). The first anchor was carefully placed posterior to the bicipital groove and just lateral to the articular margin of the humeral head, along the lateral edge of the greater tuberosity. The second anchor was placed $5 \mathrm{~mm}$ posterior to the first, and also lateral to the articular margin. One limb of the suture was passed through the tendon, $3 \mathrm{~mm}$ medial to the free edge; the other limb was passed $3 \mathrm{~mm}$ away, in an anteroposterior direction, from the first limb. This technique was then repeated for the other anchor. Each suture was then tied in a horizontal mattress configuration with a sliding double halfhitch knot, followed by alternating simple half-hitches, to a total of five throws (Fig. 3a).

Double-row anchor repair (group 2).This repair technique used a total of four suture anchors. The medial row of two anchors was placed in the same manner as described for the standard single-row anchor repair and followed by the same suturing method and placement. The lateral row of two anchors was inserted $5 \mathrm{~mm}$ lateral to the medial row, with the sutures placed through the tendon in a simple configuration. Each suture was then tied with a sliding double half-hitch knot, followed by alternating simple half-hitches, to a total of five throws (Fig. 3b).

In both groups the fascia of the retracted muscles and the skin were sutured in separate planes. Neither post-operative immobilisation nor any restrictions on activity were applied to any rabbit. Buprenorphine, $0.02 \mathrm{mg} / \mathrm{kg}$, was administered subcutaneously for post-operative pain control.

Collection of specimens. The rabbits were killed by an intravenous overdose of sodium pentobarbital $(100 \mathrm{mg} / \mathrm{kg})$ immediately after surgery, and at the fourth and eighth weeks. The supraspinatus muscle, with a small part of the scapular blade attached, and the proximal half of the humerus, were removed en bloc from both shoulders of each rabbit. The site of suture was inspected, and specimens 


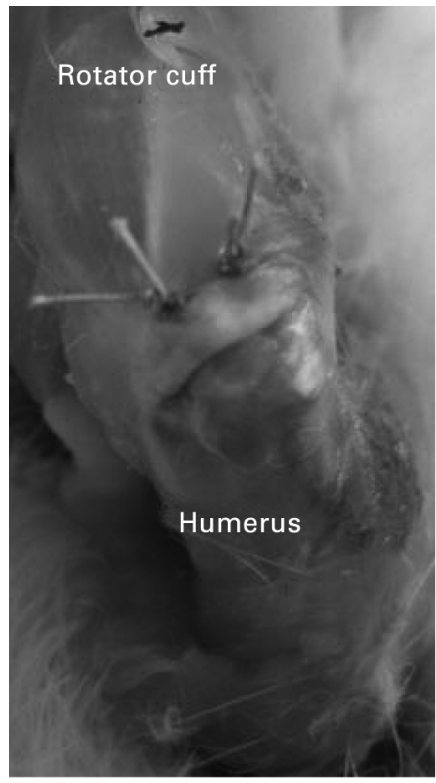

Fig. 3a

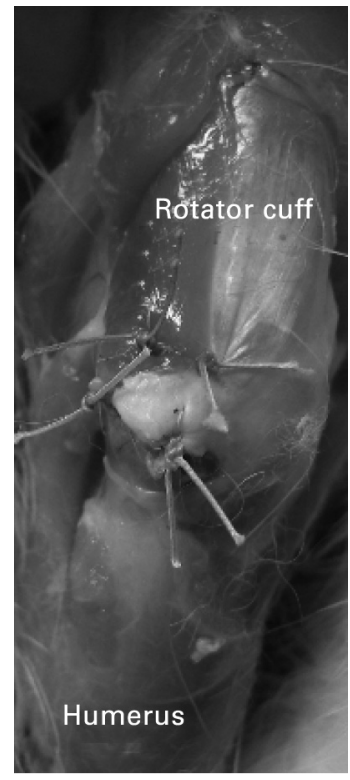

Fig. 3b
Photographs showing a) standard single-row anchor repair (group 1), and (b) double-row anchor repair (group 2).

with macroscopic evidence of dehiscence comprising a gap exceeding $0.3 \mathrm{~mm}$ were eliminated. The specimens were wrapped in a saline-soaked cloth and tested on the same day.

The rationale for the choice of four and eight weeks was based on clinical experience with humans and studies of ligament and tendon healing that have demonstrated ongoing healing throughout an eight-week period after repair. ${ }^{9-11}$

Biomechanical testing. The two repair configurations were randomised for testing. A custom-made clamp was used to grip the specimen and to mount the experimental preparation to the cross-head of a servohydraulic materials testing machine (MTS, Minneapolis, Minnesota). In order to minimise soft-tissue slippage or failure at the tendon-clamp junction, the proximal part of the supraspinatus muscletendon unit was left attached to part of the scapular spine that was sutured with no. 2, non-absorbable polyester sutures (Ethibond, Ethicon Inc., Somerville, New Jersey) and placed in a modified clamp. The humerus was rigidly secured with resin in a metal fixture, and positioned at an angle of $135^{\circ}$ to the long axis of the tendon to model the physiological pull of the supraspinatus tendon (Fig. 4). Care was taken to ensure equal and symmetrical tension on the tendon before clamping. When the specimen was mounted securely, reflective paint was used to mark the experimental set-up to allow video digitised recording.

Cyclic loading test. A cyclic loading test was performed to evaluate the performance of the repair of the rotator cuff. A 5 $\mathrm{N}$ preload was applied to pre-tension the specimen. The tendon was then cyclically loaded from $5 \mathrm{~N}$ to $30 \mathrm{~N}$ at $0.25 \mathrm{~Hz}$ for 50 cycles. The $30 \mathrm{~N}$ upper limit was chosen to approximate one-half of the ultimate loads of the weakest specimen, as determined in preliminary experiments. In the same way, 50 cycles were chosen because preliminary studies had shown that the cyclical behaviour stabilised after 40 cycles.

Load-to-failure tensile test. Following cyclical loading, each tendon specimen was loaded to failure under displacement control at a rate of $1 \mathrm{~mm} / \mathrm{s}$. Load (in Newtons) versus displacement (in millimetres) was recorded until failure. Ultimate tensile load was defined as the peak force.

Histological assessments. After biomechanical testing, all specimens were fixed in $10 \%$ neutralised formalin for one week, decalcified with formic acid for two weeks, and embedded in paraffin. Serial sections $1 \mathrm{~mm}$ apart at the tendon-bone junction were prepared from medial to the most lateral insertion of the tendon. All sections were stained with haematoxylin and eosin. The histological evaluation consisted of a qualitative assessment of the integrity of the tendon insertion site, the degree of collagenous ingrowth, and the contribution of periosteal fibres to the organisation of the tendinous attachment. In addition, the area of the tendon that healed to bone at eight weeks was evaluated by determining the number of histological sections that showed evidence of healing at the tendon-bone interface.

Statistical evaluation. The SPSS 10.0 software for Windows (SPSS Inc., Chicago, Illinois) was used to record the data and for statistical analysis. The effect of intervention (single row, double row or control) was compared for each outcome measure using the Kruskal-Wallis test. Post hoc analysis of statistically significant comparisons was conducted with multiple Mann-Whitney Utests. A p-value of 0.05 was set as the level of significance. 
Table I. Results of the cyclic and load to failure tests (mean; SD)

\begin{tabular}{lcl}
\hline & Ultimate tensile load (N) & Axial displacement (mm) \\
\hline Week 0 & $17.13(1.1)$ & $0.93(0.3)$ \\
$\quad$ Intact & $5.05(1.0)$ & $1.46(0.7)$ \\
Single row & $8.99(1.5)$ & $1.24(0.3)$ \\
Double row & & \\
Week 4 & $7.45(1.3)$ & $1.41(0.2)$ \\
$\quad$ Single row & $10.34(0.9)$ & $1.40(0.8)$ \\
Double row & & \\
& & $1.35(0.7)$ \\
Week 8 & $9.75(1.6)$ & $1.34(0.9)$ \\
$\quad$ Single row & $13.27(0.9)$ & \\
Double row &
\end{tabular}

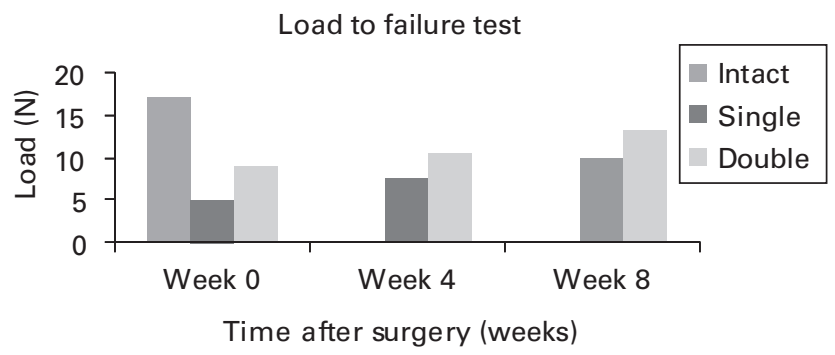

Fig. 5

Histogram of load to failure test.

\section{Results}

In all, four animals were excluded and replaced by new ones: one had a deep wound infection (double row), one had a fracture of the surgical neck of the humerus during the test (double row), and two had a wide dehiscence of the tendon repair (both single row).

Biomechanical evaluation. Failure of all the remaining specimens occurred at the site of the bone-tendon repair. The results of the cyclic and load to failure tests are presented in Table I and Figure 5.

The cyclic loading test revealed no significant difference $(\mathrm{p}=0.167$, Kruskal-Wallis) among the groups (group 1, group 2 and controls) with regard to axial displacement for all stages (week 0 , week 4 and week 8). When the group 1 mean load to failure was compared with that of group 2, ultimate tensile strength was significantly higher than in group 1 immediately after surgery $(\mathrm{p}=0.002$, Mann-Whitney $\mathrm{U}$ test $)$ and at four $(\mathrm{p}=0.001$, Mann-Whitney $\mathrm{U}$ test $)$ and eight weeks $(\mathrm{p}=0.004$, Mann-Whitney $\mathrm{U}$ test $)$ post-operatively $(\mathrm{p}<0.05)$ for all.

In addition, the mean tensile strength of group 1 (single row) increased at each interval and the differences were statistically significant $(\mathrm{p}=0.006$ for week 0 to week 4 interval; $\mathrm{p}=0.002$ for week 0 to week 8 interval; $\mathrm{p}=0.001$ for week 4 to week 8 interval, all Mann-
Whitney U test). Similarly the mean tensile strength of group 2 (double row) increased at each interval, and these differences were also significant $(\mathrm{p}=0.003$ for week 0 to week 4 interval; $\mathrm{p}=0.007$ for week 0 to week 8 interval; $\mathrm{p}=0.002$ for week 4 to week 8 interval, all MannWhitney U test). When the groups 1 and 2 loads to failure were compared with those of the intact control group, that of both groups 1 and 2 remained lower than that of the control group at all intervals, and this was also statistically significant (group $1 \mathrm{p}=0.001$ for week $0, \mathrm{p}=0.003$ for week 4 and $\mathrm{p}=0.004$ for week 8 ; group $2 \mathrm{p}=0.003$ for week $0, p=0.001$ for week 4 and $p=0.007$ for week 8, all Mann-Whitney U test).

Histological findings. The tendon stumps in both groups were closely approximated to the bone at the attachment site at four weeks. However, there were discontinuous areas at the bone-tendon interface. There was an irregular zone of oedema between the collagen bundles, with granulation tissue present. The short collagen fibrils of the woven bone were extended in a disorganised, irregular fashion into the tendon attachment (Fig. 6).

In both groups 1 and 2 the tendon was closely approximated to the bone, with the development of perpendicularly-aligned fibres within newly-formed woven bone at eight weeks. The oedema found in the four-week groups had resolved, and the tendon appeared compact, with longitudinal vessels and mature fibrocytes (Fig. 7).

When the number of histological sections with a healed tendon-bone interface was evaluated at eight weeks, the mean number of sections with healed tendon was 2.4 ( 2 to 3 ) in single-row and 3.8 ( 3 to 4 ) in the double-row groups. This difference was statistically significant $(\mathrm{p}<0.012$, Mann-Whitney U test).

\section{Discussion}

The goal of rotator cuff repair is to restore the continuity of the cuff tissue to the humerus in order to allow healing of the tendon-to-bone. ${ }^{12-16}$ It has been shown that patients in whom the repaired rotator cuff remained intact had better functional outcome, ${ }^{17,18}$ but high rates of persistent 


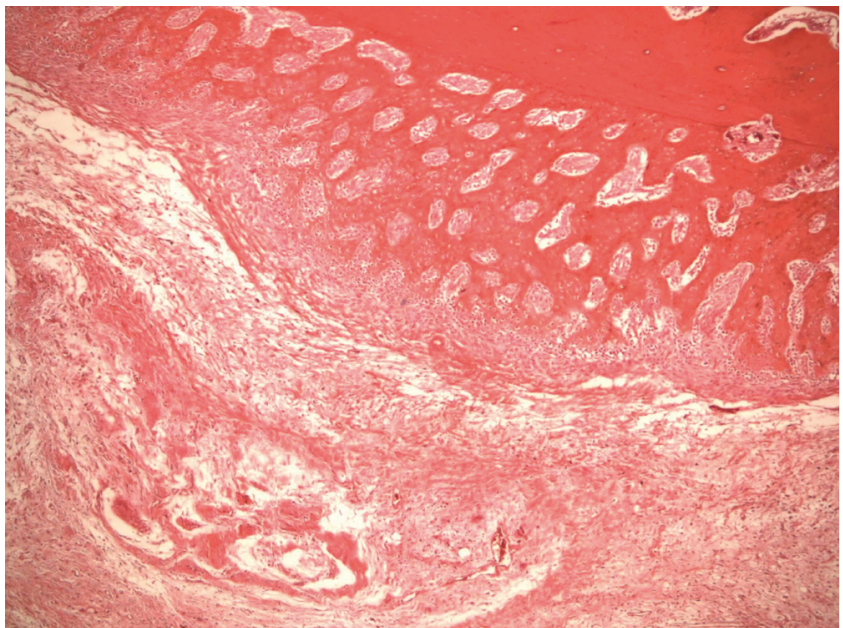

Fig. 6a

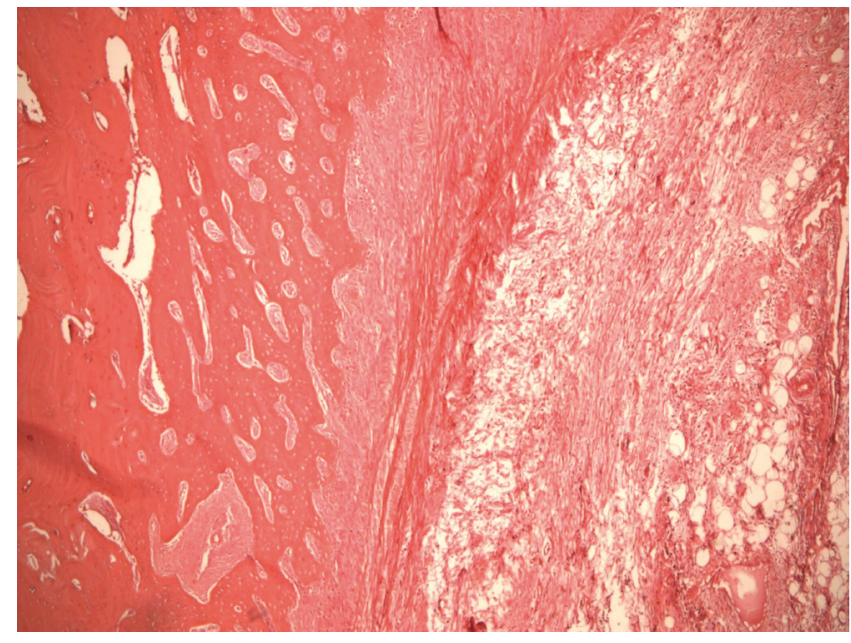

Fig. 6b

Photomicrographs showing a) the single-row and b) the double-row technique at the fourth week. Haemataxylin and eosin, magnification $\times 40$.

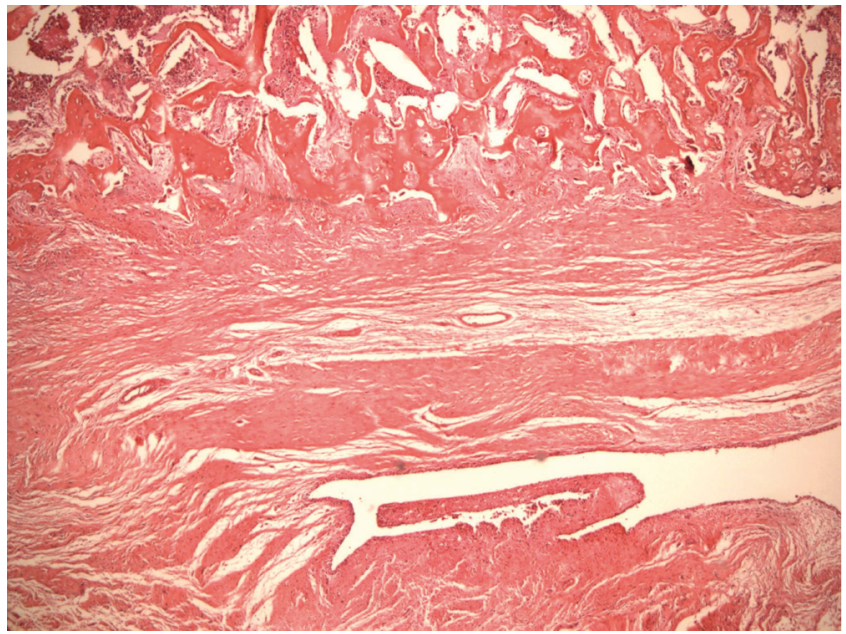

Fig. 7b

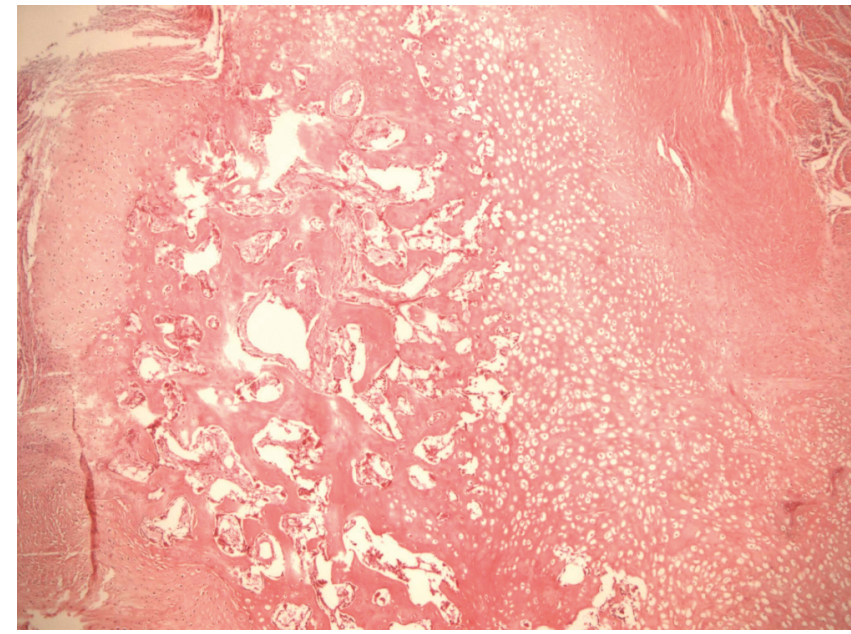

Fig. 7b

Photomicrographs showing a) the single-row and b) the double-row technique at the eighth week. Haematoxylin and eosin, magnification $\times 40$.

defects are still the most commonly reported complication after rotator cuff repair. ${ }^{19}$ The technique of the repair may affect the potential for tendon-bone healing. The doublerow fixation technique creates significantly more contact and greater overall pressure distribution over a defined area than either the transosseous suture technique or the single-row suture anchor technique. ${ }^{2,4,6,20}$

Numerous investigators have described the biomechanical properties of rotator cuff tendons after reimplantation. ${ }^{9-11}$ Miyahara et $\mathrm{al}^{11}$ studied the mechanical strength following reinsertion of the supraspinatus in dogs, and reported that the load at failure was $29.8 \%$ at two weeks, $62.5 \%$ at six weeks, and $82.5 \%$ at 24 weeks compared with controls. Uhthoff et $\mathrm{al}^{9}$ showed that return of strength of the supraspinatus tendon in rabbits was evident eight weeks after surgical reattachment into a bony trough, and that the fibrocartilaginous enthesis was normal in over half the specimens at 12 weeks. In contrast, Gerber et $\mathrm{al}^{10}$ found that the ultimate failure strength of the reattached infraspinatus in sheep was $30 \%$ at six weeks, $52 \%$ at three months, and $81 \%$ at six months. However, these investigators did not compare the effect of different repair techniques on the biomechanical properties of reimplanted tendons. The load to failure results of both operated groups in our study remained significantly lower than in the control group. During the study period the tendon-bone unit of the operated shoulder sustained a mean peak load at failure of only $57 \%$ of controls in group 1 and $77.5 \%$ of controls in group 2 at eight weeks postoperatively. The data from the present study indicate incomplete mechanical recovery even eight weeks after surgery in both groups. 
Few studies have evaluated the relationship between the attachment of the tendon to a bone surface and healing. ${ }^{20}$ Healing occurs through bone ingrowth into the fibrovascular interface tissue that forms between tendon and bone. ${ }^{20-24}$ Mechanical load has been found to be important for connective tissue healing, and probably plays a critical role in the reestablishment of a tendon-to-bone attachment. ${ }^{20,25,26} \mathrm{St}$ Pierre et $\mathrm{al}^{27}$ used a goat infraspinatus tendon-to-bone repair model to compare tendon healing to cortical bone with healing in a cancellous trough. It was concluded that the tendonhealing process was equivalent whether the tendon attachment was established between cortical or cancellous bone, as long as close apposition was maintained until collagen interdigitation occurs. Fealy et $\mathrm{al}^{20}$ studied the effect of contact area between tendon and bone on the ultimate pull-out strength of a repaired tendon. They found that increasing the number of suture anchors and the area of contact made between the tendon and bone at the site of repair, increased the load to failure of the repaired tendon at both four and eight weeks post-operatively by less than $10 \%$ at both intervals without reaching statistical significance. However, no histological evaluation of the specimens was performed. We found an approximately $43 \%, 27 \%$ and $26 \%$ gain in load to failure of a repaired rotator cuff tendon using a double-row method compared with a single-row method immediately post-operatively and at the fourth and eighth weeks, respectively. These increases were statistically significant at all stages. However, the cyclic loading test did not demonstrate any significant difference among the groups with regard to axial displacement.

We found no qualitative histological difference between the groups in terms of the tendon-healing process. The load to failure of the double-row group was significantly higher than that of the single-row group initially and at four weeks, and appeared to be related to the number of anchors used. However, in the double-row group the increase of load to failure at eight weeks seemed to be mostly related to the larger surface area of tendon that healed to bone.

Our study had some limitations. First, the animal model may not accurately reflect the healing pattern in humans. Secondly, an acute division was created which does not reflect the usual chronic tear pattern seen in the human rotator cuff. Thirdly, the repair was performed acutely, which is also a situation that rarely occurs in humans.

In conclusion, the load to failure for both operated groups remained significantly lower than in the control group. Increasing the number of suture anchors used leads to an increase in the load to failure in the immediate and four-week post-operative period. In turn, the larger number of anchors leads to an increase in surface area for tendon-to-bone healing, which produces a significant increase in the ultimate load to failure of the repaired tendon at eight weeks postoperatively.

This study was supported by the Research Fund of the Scoiety of Turkish Orthopaedics and Traumatology.
No benefits in any form have been received or will be received from a commercial party related directly or indirectly to the subject of this article.

\section{References}

1. Curtis AS, Burbank KM, Tierney JJ, Scheller AD, Curran AR. The insertional footprint of the rotator cuff: an anatomic study. Arthroscopy 2006;22:609.

2. Apreleva M, Ozbaydar M, Fitzgibbons PG, Warner JJ. Rotator cuff tears: the effect of the reconstruction method on three-dimensional repair site area. Arthroscopy 2002;18:519-26

3. Mazzocca AD, Millet PJ, Guanche CA, Santangelo SA, Arciero RA. Arthroscopic single-row atrophy and fat accumulation of the supraspinatus: an experimental study in rabbits. Orthop Res 2002;20:357-63.

4. Meier SW, Meier JD. Rotator cuff repair: the effect of double-row fixation on threedimensional repair site. J Shoulder Elbow Surg 2006;15:691-6.

5. Ma CB, Comerford L, Wilson J, Puttlitz CM. Biomechanical evaluation of arthroscopic rotator cuff repairs: double-row compared with single-row fixation. J Bone Joint Surg [Am] 2006;88-A:403-10.

6. Meier SW, Meier JD. The effect of double-row fixation on initial repair strength in rotator cuff repair: a biomechanical study. Arthroscopy 2006;22:1168-73.

7. Koike Y, Trudel G, Curran D, Uhthoff HK. Delay of supraspinatus repair by up to 12 weeks does not impair enthesis formation: a quantitative histologic study in rabbits. $J$ Orthop Res 2006:24:202-10.

8. Matsumoto F, Uhthoff HK, Trudel G, Loehr FJ. Delayed tendon reattachment does not reverse atrophy and fat accumulation of the supraspinatus: an experimental study in rabbits. J Orthop Res 2002;20:357-63.

9. Uhthoff HK, Seki M, Backman DS, et al. Tensile strength of the supraspinatus after reimplantation into a bony trough: an experimental study in rabbits. J Shoulder Elbow Surg 2002;11:504-9.

10. Gerber C, Schneeberger AG, Perren SM, Nyffeler RW. Experimental rotator cuff repair: a preliminary study. J Bone Joint Surg [Am] 1999;81-A:1281-90.

11. Miyahara H, Takagishi K, Arita C, et al. A morphologic and biomechanical study on the healing of the repaired rotator cuff insertion in dogs: a preliminary report. In: Post M, Morrey BF, Hawkins RJ, eds. Surgery of the shoulder. St. Louis: Mosby, 1990:224-7.

12. ElIman H, Kay SP, Wirth M. Arthroscopic treatment of full-thickness rotator cuff tears: 2- to 7-year follow-up study. Arthroscopy 1993;9:195-200.

13. Gartsman GM, Khan M, Hammerman SM. Arthroscopic repair of full-thickness tears of the rotator cuff. J Bone Joint Surg [Am] 1998;80-A:832-40.

14. Ianotti JP. Full-thickness rotator cuff tears: factors affecting surgical outcome. J Am Acad Orthop Surg 1994;2:87-95.

15. Tauro JC. Arthroscopic rotator cuff repair: analysis of technique and results at 2- and 3year follow-up. Arthroscopy 1998;14:45-51.

16. Warner JJ, Tétreault P, Lehtinen J, Zurakowski D. Arthroscopic versus mini-open rotator cuff repair: a cohort comparison study. Arthroscopy 2005;21:328-32

17. Harryman DT 2nd, Mack LA, Wang KY, et al. Repairs of the rotator cuff: correlation of functional results with integrity of the cuff. J Bone Joint Surg [Am] 1991;73-A:982-9.

18. Lundberg BJ. The correlation of clinical evaluation with operative findings and prognosis in rotator cuff rupture. In: Bayley I, Kessel L, eds. Shoulder surgery. Berlin: Springer-Verlag, 1982:35-8.

19. Galatz LM, Ball CM, Teefey SA, et al. The outcome and repair integrity of completely arthroscopically repaired large and massive rotator cuff tears. J Bone Joint Surg [Am] 2004;86-A:219-24.

20. Fealy S, Rodeo SA, MacGillivray JD, et al. Biomechanical evaluation of the relation between number of suture anchors and strength of the bone-tendon interface in a goat rotator cuff model. Arthroscopy 2006;22:595-602.

21. Arnoczky SP, Torzilli PA, Warren RF, Allen AA. Biologic fixation of ligament prostheses and augmentations: an elevation of bone ingrowth in the dog. Am J Sports Med 1988;16:106-12

22. Berlet GC, Johnson JA, Milne AD, Patterson SD, King GJ. Distal biceps brachii tendon repair: an in vitro biomechanical study of tendon reattachment. Am J Sports Med 1998;26:428-32.

23. Forward AD, Cowan RJ. Tendon suture to bone: an experimental investigation in rabbits. J Bone Joint Surg [Am] 1963;45-A:807-23

24. Gao J, Wei X, Messner K. Healing of the anterior attachment of the rabbit meniscus to bone. Clin Orthop 1998;348:246-58.

25. Robertson DB, Daniel DM, Biden E. Soft tissue fixation to bone. Am J Sport Med 1986;14:398-403.

26. Shino K, Kawasaki T, Hirose $\mathbf{H}$, et al. Replacement of the anterior cruciate ligament by an allogeneic tendon graft: an experimental study in the dog. J Bone Joint Surg $[\mathrm{Br}]$ 1984;66-B:672-81.

27. St Pierre $\mathbf{P}$, Olsen EJ, Elliott JJ, et al. Tendon-healing to cortical bone compared with healing to a cancellous trough: a biomechanical and histological evaluation in goats. J Bone Joint Surg [Am] 1995;77-A:1858-66. 